\title{
Commentary for the article: MicroRNA-1246 regulates proliferation, invasion and differentiation in human vascular smooth muscle cell by targeting cystic fibrosis transmembrane conductance regulator (CFTR)
}

\author{
Marcelo Roberto $\mathrm{Choi}^{1,2}$ (1) \\ Received: 29 December 2020 /Revised: 29 December 2020 / Accepted: 4 January 2021 / Published online: 16 January 2021 \\ (C) The Author(s), under exclusive licence to Springer-Verlag GmbH, DE part of Springer Nature 2021
}

In this issue of Pflügers Archiv European Journal of Physiology, Pan et al. demonstrate the effects of miR-1246 in the regulation of proliferation, migration, and differentiation of vascular smooth muscle cells (VSMCs) [6]. By using human VSMC cell lines and molecular, cellular and functional approaches, the authors demonstrated that miR-1246 actions on VSMCs are exert through downregulation of cystic fibrosis transmembrane conductance regulator (CFTR) protein. Atherosclerosis is a chronic low-grade inflammatory process that involves large and medium-caliber arteries and is one of the leading causes of morbidity and mortality worldwide. Therefore, it is essential to advance in the knowledge of the cellular and molecular mechanisms that lead to its development and complication given its high impact on health $[1,4]$. VSMCs dysfunction represent a key step in the pathophysiology of atherosclerotic lesion [9], turning the comprehension of the mechanisms that lead to VSMCs dysfunction an area of great interest since they can offer an alternative for the development of new therapeutic strategies.

Recent findings support the fact that miRNAs are involved in different pathological process of VSMCs by stimulating a high rate of proliferation, migratory capacity, apoptosis, calcification, aging, and switch their contractile differentiation to

This article is a commentary to the original article https://doi.org/10.1007/ s00424-020-02498-8

Marcelo Roberto Choi

marcelinkchoi@yahoo.com.ar

1 Universidad de Buenos Aires, Facultad de Farmacia y Bioquímica, Cátedra de Anatomía e Histología, Buenos Aires, Argentina

2 Universidad de Buenos Aires, CONICET, Instituto Alberto C. Taquini de Investigaciones en Medicina Traslacional (IATIMET), Buenos Aires, Argentina a secretory phenotype, which leads to formation of a neointima with excessive extracellular matrix deposition and inflammation that contributes to progressive damage during atherosclerosis (Fig. 1) [1, 2, 4, 9]. Furthermore, Sun et al. [8] have shown in an atherosclerosis model of Apo E -/- mice fed with a western diet for 16 weeks, that miR-146b-5p protects against atherosclerosis by inhibiting VSMCs proliferation and migration. In this regard, it should be noted that some drugs like olmesartan (an Ang II AT1 receptor antagonist) are also capable of regulating miRNAs, such as miR-665, improving VSMC dysfunction [10].

The molecular targets of miRNAs involve downstream elements such as transcription factors and intracellular signaling cascades that control basic cellular processes in VSMCs. Thus, the proliferative and migratory phenotypes have been described to be induced when some miRNAs are increased, such as miR-24, miR-26a, miR-146a, miR-221, miR-222, or when other miRNAs are decreased like miR-1, miR-31, miR125b, miR-133, and miR-143 [1, 2, 4, 9]. Some of the intracellular targets of miRNA can affect VSMCs proliferation by regulating cell cycle molecules and apoptosis pathway such as kinase-dependent cyclins. Additionally, the synthetic and secretory phenotypes can be stimulated by increasing the expression of miR-21, miR-26a, and miR-34a or by decreasing the expression of miR-29b, miR-31, miR-145, and miR-205 $[1,2,4,9]$. The present study of Pan et al. now adds a new player, miR-1246, to the list of miRNAs involved in VSMC dysfunction [6]. Recently, miRNA-1246 has gained relevance as a genomic regulator that promotes proliferation and invasion in tumorigenesis [7]. The authors also demonstrate the association of the chloride channel CFTR transporter as the molecular target of miR-1246. The CFTR transporter acts as a chloride channel (cAMP dependent) and it has been reported to be involved in the regulation of $\mathrm{pH}$, cell volume, and proliferation and migration [5]. Previous studies have shown that 
miR-34b, miR-30b, miR-26a, miR-135a

miR-125b, miR-204, miR-182, miR-29b
miR-32, miR-34a, miR-221, miR-222

miR-134, miR-223
miR-21, miR-26a

miR-146a, miR-24

miR-221, miR-147b

miR-222, miR-130a

miR-208

miR-21, miR-221

miR-26a, miR-222

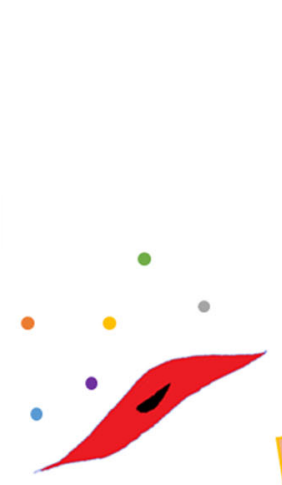

miR-15a, miR-34a

miR-34c, miR-30e

miR-132

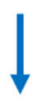

\section{Apoptosis}

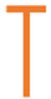

miR-21, miR-25

miR-26a, Mir-92a

miR-106a, miR-125b

miR-133a, miR-210,

miR-296, miR-4632

miR-146a

$$
\text { Calcification }
$$

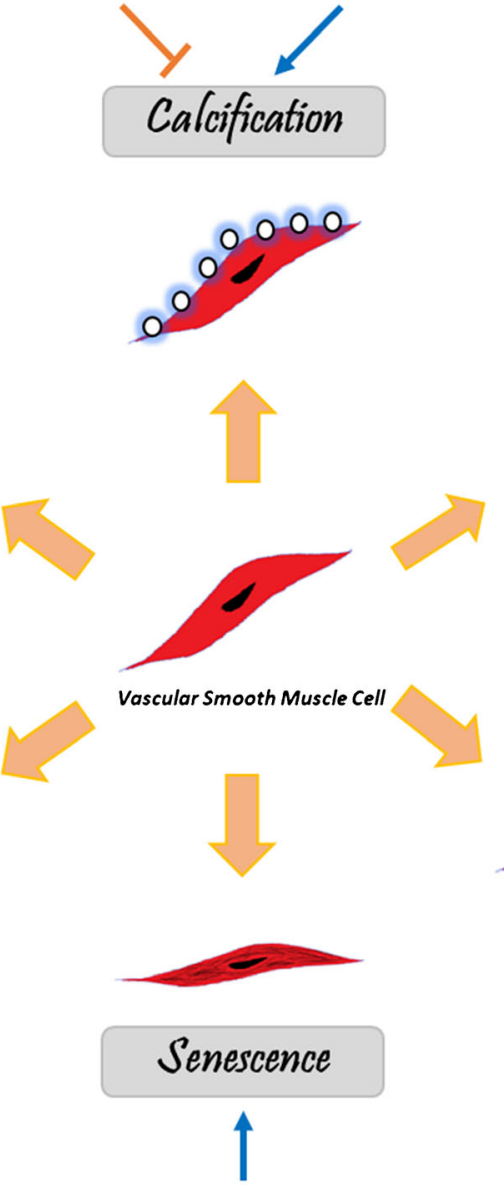

miR-21

miR-146a

miR-34a

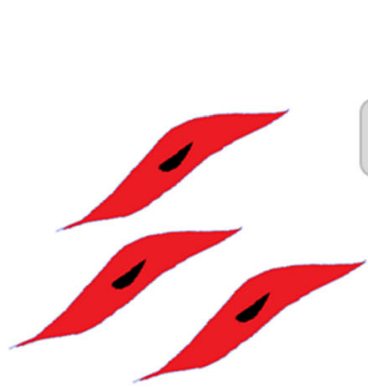

Proliferation

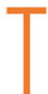

miR-34a, miR-1

miR-125b, miR-143

miR-365b, miR-31

miR-145, miR-133

miR-15a, miR-152

miR-155, miR-181a

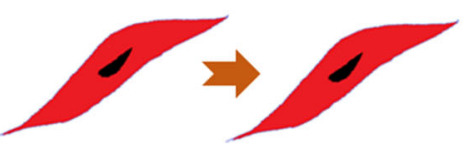

Migration

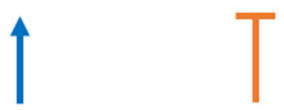

miR-223, miR-24

miR-146a, miR-221

miR-147b, miR-222

miR-133, miR-29b

miR-26a
miR-125b, miR-1

miR-145, miR-133

miR-365b, miR-31

miR-143, miR-181a

miR-195, miR-638

Fig. 1 The multiple roles of miRNAs in VSMCs in the pathogenesis of atherosclerosis. Solid arrow indicates stimulation and T-line indicates inhibition

the CFTR channel is not only involved in the vascular remodeling of cerebral arteries, but it also protects against vascular inflammation and atherogenesis in apo $\mathrm{E}$ deficient mice, by decreasing NFkB levels and MAPK activity $[9,10]$. The activation of this CFTR channel, not only inhibits oxidative stress, but also prevents the development of arterial hypertension by facilitating VSMCs relaxation $[3,11]$.

Together, all this evidence highlights the importance of miRNAs as key regulators of vascular function, thus exposing an intricate network of subcellular interaction typical of a complex pathological process as atherosclerosis. Meanwhile, further investigations will be required to elucidate a possible pharmacological modulation of miRNAs as a promising candidate for a new therapeutic strategy for atherosclerosis.

\section{Compliance with ethical standards}

Conflict of interest The authors declare he has no conflict of interest.

\section{References}

1. Basatemur GL, Jørgensen HF, Clarke MCH, Bennett MR, Mallat Z (2019) Vascular smooth muscle cells in atherosclerosis. Nat Rev Cardiol 16(12):727-744

2. Churov A, Summerhill V, Grechko A, Orekhova V, Orekhov A (2019) MicroRNAs as potential biomarkers in atherosclerosis. Int J Mol Sci 20(22):5547

3. Hübner CA, Schroeder BC, Ehmke H (2015) Regulation of vascular tone and arterial blood pressure: role of chloride transport in vascular smooth muscle. Pflügers Arch 467(3):605-614

4. Li L, Li Y, Tang C (2019) The role of microRNAs in the involvement of vascular smooth muscle cells in the development of atherosclerosis. Cell Biol Int 43(10):1102-1112

5. Li Z, Shen Z, Xue H, Cheng S, Ji Q, Liu Y, Yang X (2017) CFTR protects against vascular inflammation and atherogenesis in apolipoprotein E-deficient mice. Biosci Rep 37(4):BSR20170680

6. Pan D, Liu G, Li B et al (2021) MicroRNA-1246 regulates proliferation, invasion, and differentiation in human vascular smooth muscle cells by targeting cystic fibrosis transmembrane conductance regulator (CFTR). Pflugers Arch. https://doi.org/10.1007/ s00424-020-02498-8 
7. Sakha S, Muramatsu T, Ueda K, Inazawa J (2016) Exosomal microRNA miR-1246 induces cell motility and invasion through the regulation of DENND2D in oral squamous cell carcinoma. Sci Rep 6:38750

8. Sun D, Xiang G, Wang J, Li Y, Mei S, Ding H, Yan J (2020) miRNA $146 \mathrm{~b}-5 \mathrm{p}$ protects against atherosclerosis by inhibiting vascular smooth muscle cell proliferation and migration. Epigenomics. https://doi.org/10.2217/epi-2020-0155

9. Yu X, Li Z (2014) MicroRNAs regulate vascular smooth muscle cell functions in atherosclerosis. Int J Mol Med 34(4):923-933
10. Zhang Y, Liang Q, Zhang Y, Hong L, Lei D, Zhang L (2020) Olmesartan alleviates bleomycin-mediated vascular smooth muscle cell senescence via the miR-665/SDC1 axis. Am J Transl Res 12(9):5205-5220

11. Zhang Y, Zhang H, Duan DD (2013) Chloride channels in stroke. Acta Pharmacol Sin 34(1):17-23

Publisher's note Springer Nature remains neutral with regard to jurisdictional claims in published maps and institutional affiliations. 